\title{
Disparitions, coercition et violence symbolique
}

\section{Didier Bigo}

\section{OpenEdition \\ Journals}

\section{Édition électronique}

URL : http://journals.openedition.org/conflits/181

DOI : $10.4000 /$ conflits. 181

ISSN : $1777-5345$

Éditeur :

CCLS - Centre d'études sur les conflits lilberté et sécurité, L'Harmattan

Édition imprimée

Date de publication : 15 mai 1994

ISSN : 1157-996X

Référence électronique

Didier Bigo, «Disparitions, coercition et violence symbolique », Cultures \& Conflits [En ligne], 13-14 | printemps-été 1994, mis en ligne le 14 mars 2006, consulté le 30 mars 2021. URL : http:// journals.openedition.org/conflits/181 ; DOI : https://doi.org/10.4000/conflits.181

Ce document a été généré automatiquement le 30 mars 2021.

Creative Commons License 


\section{Disparitions, coercition et violence symbolique}

\section{Didier Bigo}

1 Nombre de pratiques coercitives ${ }^{1}$ ont déjà fait l'objet d'analyse sociologique et historique de qualité. Qu'il s'agisse des politiques de génocide ${ }^{2}$ ou qu'il s'agisse des exactions commises par les gouvernements à l'égard des minorités ${ }^{3}$. Mais, si les politiques d'extermination systématique ou d'exécutions sommaires pour appartenance à un groupe (social, ethnique, religieux, politique...) d'une part, et les "débordements" de la guerre d'autre part, ont retenu l'attention des chercheurs, peu a été dit sur les pratiques de "disparition" d'une manière comparative. Nous pouvons certes nous appuyer sur des études historiques et politiques solides concernant l'Amérique latine des années 70 (Chili, Argentine, Brésil) ${ }^{4}$, mais qu'en est-il ailleurs ? Comment analyser les phénomènes de disparition en Afrique, au Sri Lanka, au Maroc? Que dire sur ce qui se passe actuellement et quelles différences avec ce qui se passait dans les années $1970 ?^{5}$

2 A un moment où l'on parle tant de "diplomatie préventive", à un moment où les transformations de la bipolarité ont induit de nouvelles modalités d'opérations armées pour "raison humanitaire" et où les politiques de "secret" des gouvernements en ont été modifiées, il peut être utile de s'interroger sur les stratégies de la coercition, sur leurs modalités (en particulier les disparitions), et sur leur relation avec le "contexte" international. Il y a quelques raisons de penser que la modification de ce dernier, en bousculant encore plus qu'auparavant la possibilité pour les gouvernants de se réfugier derrière la "non ingérence dans les affaires intérieures" et les autres corrélats de la souveraineté, va pousser certains gouvernements qui pratiquent à large échelle la coercition, à être plus circonspects sur leurs manières d'agir. L'éclairage par le marché humanitaire ${ }^{6}$ peut renforcer la course au "sacrifice spectaculaire" dans certaines mobilisations confessionnelles ou ethno-nationalitaires, il peut aussi, à l'inverse, renforcer les stratégies "d'invisibilisation de la violence sur les corps" pour motif de "respectabilité internationale"; stratégies de dissimulation souvent liées aux 
impératifs des acteurs locaux d'extraire des ressources (de légitimation, financières,...) du milieu international.

3 Le renforcement à l'échelle mondiale des interdépendances sociales autant qu'économiques peut les pousser à renoncer à la coercition massive, quitte à abandonner le pouvoir d'Etat ; cela peut aussi les pousser simplement à masquer encore plus qu'auparavant leurs pratiques coercitives et à avoir recours à ce que l'on nomme communément des "disparitions" forcées? ${ }^{7}$ Il est sans doute trop tôt pour analyser en détail en quoi les modifications du contexte international jouent sur le répertoire des pratiques de coercition interne mais nous reviendrons dans un autre numéro sur ce rapport entre pratiques des disparitions, jeux politiques internes et légitimation des pouvoirs sur la scène internationale. Ici nous voudrions avant tout comprendre comment ces pratiques de disparitions s'agencent et à quoi elles renvoient. Sont-elles une simple absence de connaissance et donc un phénomène momentané qui se résorberait pour l'essentiel dans les exécutions extrajudiciaires une fois les événements connus? Sont-elles un simple masque ou au contraire une stratégie spécifique à l'intérieur d'un répertoire d'action coercitive ajoutant la violence symbolique créée par l'incertitude quant au sort du "disparu", et la "complicité " objectivée des proches, à la violence physique sur le corps lui-même ? Sont-elles le produit des interactions sociales dans les sociétés où la violence se généralise et échappe à la maîtrise de ceux qui l'avaient initiée?

4 Disparaître Disparaître, c'est en premier lieu laisser tous les autres sans nouvelles. C'est créer de l'inquiétude autour de soi. C'est générer de l'angoisse. C'est souvent pousser les autres à rechercher à savoir ce qui s'est passé. Le premier mouvement dans nos sociétés est d'en appeler à la police pour faire ces recherches mais qu'en est-il lorsque l'on soupçonne la police ou d'autres corps de l'Etat d'être à l'origine de cette disparition qui ne serait donc pas volontaire? Faut-il oser continuer les recherches avec le risque de disparaître à son tour si l'on en apprend trop ? Faut-il se taire ? Faut-il constituer des groupes de recherche et se mobiliser en attirant l'attention internationale sur les pratiques du pouvoir? Faut-il se faire aider par des ONG ? Dans de nombreux cas les "personnes disparues" ont subi des arrestations, des tortures et en sont mortes. Le critère du régime politique et de l'attitude des forces de coercition est ici déterminant. Au nom de la Raison d'Etat, les gouvernants veulent masquer leurs propres pratiques et se distancier des ordres qu'ils ont donnés, en particulier dans les pays liés à la sphère occidentale (Turquie, Maroc, Syrie...). Au delà ils visent à établir un régime de vérité fondé sur le travail de méconnaissance, de dénégation de la société à l'égard des pratiques de disparition forcée. Il s'agit de faire admettre la vérité du pouvoir (et la "non existence" des disparus) aux Tiers et même aux proches. Celle-ci doit devenir inquestionnable. Pourtant, même sous les régimes les plus autoritaires, le travail de la mémoire, allié au temps, sape le processus d'invisibilisation à l'origine des disparitions. Le travail de deuil est-il si important qu'il faille risquer sa vie pour récupérer un cadavre ? La réponse fréquente des proches est oui car la souffrance vient aussi de l'incertitude générée par la disparition et du maigre espoir toujours possible d'une "erreur", d'une "libération". C'est sans doute dans ce trait de l'incertitude quant au sort du disparu comme dans le processus de méconnaissance qui l'accompagne (le pouvoir ne l'a pas voulu, c'est une erreur dans ce cas précis...) qui produit une violence symbolique très puissante que réside la spécificité des disparitions. 
5 Les disparitions : produit d'une ignorance Les disparitions, nous le verrons, ont du mal à être une catégorie de violation des droits de l'Homme "comme les autres". Elles sont l'objet de présomptions, de soupçons mais ne peuvent s'étayer justement sur la matérialité du corps de la personne disparue dans un premier temps et fréquemment, dans un second temps, elles sont l'objet de stratégies diverses, de transactions entre acteurs sur le niveau de connaissance qu'il est acceptable de "révéler" à la population8 ${ }^{8}$. Elles reposent en effet au moment de l'acte uniquement sur l'appréciation des raisons de "l'invisibilisation" du corps par le pouvoir, et sur le fait que la disparition lui serait imputable. C'est ensuite, souvent des années plus tard, que la connaissance du sort réservé à la personne les fait changer de catégorie. Elles deviennent cas de massacre collectif, de vengeances, de tortures... On comprend que nombre d'approches (y compris dans ce numéro) se refusent à distinguer les disparitions des autres pratiques coercitives. Seulement ce "bon sens" juridique et comptable a ses limites et produit des effets de méconnaissance en faisant des disparitions une simple pratique secondaire et polymorphe des autres cas de violation des droits de l'Homme.

6 Or, le pouvoir n'est jamais tant "producteur de vérité" que lorsqu'il peut choisir de rendre visible ou invisible son inscription sur les corps de ses sujets. Le discours comme pouvoir est à son point maximum d'application lorsqu'il nie l'existence de ces corps comme dans le cas des disparitions et c'est cette dimension de violence symbolique qui généralement n'est pas perçue. Les différentes ONG restent à cet égard dépendantes de cette appréciation de la "vérité" par les acteurs et il n'est pas sans intérêt de noter qu'elles adoptent une ligne de conduite "pragmatique" et "victimologique" qui les conduit à ignorer la spécificité de la disparition comme répertoire d'action ajoutant la violence symbolique à la coercition au profit d'une approche où les disparitions ne sont plus que résiduelles, sorte d'écart entre les pratiques de pouvoir et les connaissances que les autres acteurs ont de celles-ci. Les disparitions ne sont alors analysées que sous l'angle de leur relation avec les emprisonnements arbitraires, les tortures, les exécutions extrajudiciaires. Elles sont le résultat d'une ignorance des conditions de la coercition et du sort des victimes.

7 Les disparitions : stratégie "discrète" Pourtant il est sans doute possible de montrer que, pour partie des cas recensés, les disparitions renvoient à des stratégies de pouvoir dont la spécificité tient à cette violence symbolique de l'invisibilisation qui en faisant disparaître le corps ajoute par l'incertitude à la violence physique. On doit donc sans doute distinguer les dispositifs où les cas de "disparition" tiennent à l'ignorance d'événements connus beaucoup plus tard (cas de massacres collectifs lors de guerre internationale ou civile qui "disparaissent" devant l'ampleur d'autres événements, charniers découverts a posteriori), des dispositifs où la disparition apparaît comme une stratégie "discrète" visant certaines personnes plus que d'autres.

8 Dans le premier dispositif : celui des "machines guerrières", celui de la "polarisation duelle entre adversaires", celui de la "suppression de toute position de Tiers indifférent" ${ }^{\prime \prime}$, s'il existe des personnes disparues, ce n'est souvent que la résultante d'une ignorance à l'égard de l'ampleur et des formes prises par la coercition ${ }^{10}$. Lors des périodes de guerre, de guerre civile ou de combats importants entre guérilla et armée, les acteurs peuvent massacrer leurs adversaires et les populations qui sont censées les soutenir mais il n'y a pas forcément de stratégie spécifique concernant la conduite à tenir à l'égard des corps. On ne fait pas disparaître des vivants mais des cadavres. Dans certains cas on masque les charniers car ces "débordements" de la guerre en brisent les 
"lois"11, mais parfois, loin qu'il y ait secret, le groupe qui a perpétué le massacre met en scène sa propre violence et la théâtralise (Indonésie dans les années 60, Ouganda des années 70 , Guatemala dans les années 80 , Sud Soudan, Timor, Pérou, Tchad, voire Bosnie). Il s'agit de marquer la prise de territoire, de provoquer la terreur par la marque des supplices sur les corps.

En revanche dans le second dispositif (celui dans lequel un pouvoir cherche à éliminer des opposants souvent désarmés et sur un territoire qu'il contrôle déjà sans réelle remise en cause) il y a une stratégie spécifique de quadrillage sociétal par le gouvernement ou des agences plus ou moins officielles visant par la "disparition" de certaines personnes à créer une "insécurité" permanente, à distendre les liens sociaux, tout en tenant compte des risques de réaction internationale (et interne) si cette politique coercitive était "trop" voyante. L'invisibilité est alors une stratégie non une résultante ${ }^{12}$. Il y a un projet réfléchi de faire disparaître et de créer une incertitude chez les proches du disparu et chez ceux qui pourraient compatir (intellectuels, camarades...) mais de le faire le plus secrètement possible afin de tenir compte des jeux d'alliance à l'échelle interne et internationale ${ }^{13}$. Il y a une logique spécifique qui se met en place "d'invisibilisation". C'est une logique de "secret" ou plutôt de "discrétion". En effet cette stratégie peut être dite discrète tant au sens politique (secret, masque) qu'au sens mathématique (sériel, discontinu). Elle est discrète plus que secréte en ce qu'elle dissimule l'ampleur des pratiques mais laisse parfois jouer ou même provoque les rumeurs les plus folles. Elle masque les corps mais pour mieux imposer silence aux proches et les contraindre à transiger en contrepartie de nouvelles, d'espoirs... elle est discrète au sens de sériel en ce qu'elle s'oppose aux logiques de génocide et de politicide qui visent au contraire à détruire en continu, intégralement.

Le dispositif qui se met en place nie en effet les corps individuels sur lesquels il entend s'appliquer : on ne doit ni les voir, ni en parler, mais son point d'application réel est moins l'humanité de l'individu que la relation qu'il entretient aux autres. Ce dispositif ne vise pas à l'élimination de tous comme dans le génocide ou même de toute une minorité particulière mais à l'invisibilisation sociale des personnes pouvant rassembler, coaliser des groupes. Il vise plutôt à casser le corps social en le discrétisant, en le frappant là où le pouvoir pense qu'il s'articule. La stratégie de disparition vise à briser des réseaux sociaux. L'individu, le village, le groupe social, politique ou religieux qui s'oppose à la production de vérité du pouvoir doit disparaître sans laisser de trace. Les disparus n'ont pas d'existence, à la limite ils n'en ont jamais eu maintenant que le pouvoir veut en décider ainsi. Par l'invisibilisation de certains, et par la tentative d'intimidation des autres, il s'agit de casser l'émergence d'un espace politique en détruisant les relations sociales, en atomisant les groupes. Argentine, Uruguay et Chili des généraux en ont été des cas exemplaires. Mais c'est aussi le cas des Philippines, de l'Inde, du Mexique, de l'Afrique du Sud, de la Syrie, du Maroc, de l'Arabie Saoudite, de la Turquie, de l'Algérie ou de l'Egypte... A chaque fois ces régimes ont une caractéristique commune au delà de leurs profondes différences. Ils veulent éliminer des opposants mais sans que cela soulève des protestations internationales gênant les relations inter-gouvernementales avec les pays occidentaux.

11 Cela explique aussi que dans ce dispositif de pouvoir, les gouvernants n'usent pas simplement des forces armées habituelles (comme c'est le cas lors des massacres collectifs dans les affrontements de période de guerre). Ils préfèrent souvent susciter au sein des forces armées ou de police, voire dans des partis politiques extrémistes, des 
organisations secrètes (escadrons de la mort) qui frapperont pour eux : enlèvements, rafles, camps de détention non officiels... Cela leur permet en cas de réaction internationale trop vive, et s'il est impossible de nier en bloc, de pouvoir rejeter la faute sur ces éléments "incontrôlés". Mais sinon ils les soutiennent, les couvrent et surtout élaborent des rhétoriques justifiant ces pratiques (doctrine de la sécurité nationale, danger communiste ou intégriste ou séparatiste, lutte antiterroriste...). Ils font admettre, même a posteriori, que la "sale guerre" avait ses raisons, que les arrestations arbitraires, les tortures et les disparitions s'expliquaient en raison du péril interne ou externe. Ce sont aussi les premiers régimes à contrer par leur appareil de propagande le travail des organisations humanitaires ${ }^{14}$ et à faire jouer les liens de lobbying qui les lient souvent avec des franges des appareils politiques des Etats de droit ${ }^{15}$.

Cette stratégie initiée par le pouvoir n'est pourtant jamais pleinement réalisée car, si le pouvoir par son atteinte aux identités, aux corps cherche à rendre invisible son empreinte, il crée aussi dans le jeu des interactions les conditions d'une visibilité du processus d'invisibilisation comme ces femmes dansant avec leurs maris disparus sur la place de Mai en Argentine et les rendant encore plus visible par leur invisibilité même, par la présence de leur absence. La variable temporelle est ici cruciale. Dans l'instant le programme de vérité du pouvoir s'impose sans doute en s'appuyant sur la complicité objectivée d'une société (interne et internationale) qui refuse de voir cette invisibilisation pour continuer à vivre comme avant. Mais dans la durée, ce pouvoir symbolique peut être remis en cause. L'analyse des situations de "transition" politique auxquelles nous consacrerons notre prochain numéro montre à la fois l'impossibilité de la "réalisation" de la volonté de pouvoir/savoir et en même temps ses capacités à exiger des acteurs des transitions, même après coup, que tout ne soit pas dit. Lorsque les proches des disparus redeviennent acteurs politiques, lorsque le travail de mémoire s'élabore pour faire échec à la volonté de silence et d'invisibilité du pouvoir, ils font lire et voir ce que tout le monde savait et disait mais ne pouvait pas exprimer viva voce.

Disparition et acteurs en concurrence Par ailleurs parfois cette stratégie se heurte à la possible autonomisation des groupes clandestins qui peuvent prolonger la violence bien au delà de ce qui était voulu par les dirigeants. La dynamique d'interaction peut engendrer le passage d'une stratégie réfléchie et sélective à des logiques de vengeance ou à une généralisation des pratiques de disparition pour "supprimer les témoins". Dans des dispositifs de pouvoir où ce dernier est moins sûr de sa propre force ou moins sûr de ses légitimations, où le quadrillage est énoncé mais où les pratiques de contrôle ne s'appliquent pas avec la même intensité, les disparitions n'ont pas forcément la même signification. Elles ne sont pas simples résultantes mais elles ne sont pas non plus stratégie délibérée d'un acteur surpuissant et imposant le cadre de la relation de pouvoir, elles proviennent au contraire des luttes entre acteurs multiples pour l'imposition de ce cadre et dans ce cadre. Qu'il s'agisse du Salvador, du Sahara Occidental, du Guatemala, de la Colombie ou du Sri Lanka, on observe cette dynamique. La disparition n'est donc pas réductible dans tous les cas de figures à une "technologie de pouvoir d'Etat", à un répertoire d'action coercitive, elle est aussi "produit" de la relation. Les pratiques de disparition ne relèvent donc pas toujours des projets stratégiques des dirigeants, de leur manière d'objectiver le peuple, de leurs énonciations doctrinaires sur le figure de l'ennemi (le terroriste, le subversif, l'intégriste...), elles peuvent avoir lieu en dehors d'un projet préalable. Les appareils policiers ou paramilitaires qui commencent par des disparitions "sérielles" s'inquiètent par exemple d'être reconnus parce qu'ils anticipent un risque de renversement du 
pouvoir en place et s'engagent dès lors dans une "chasse aux témoins" dont nombre d'enquêtes d'Amnistie montrent l'importance en terme de motivation. Une personne était visée mais finalement on s'en prend à sa famille, à ses proches et par extension à son lignage ethnique ou à tous ceux qui fréquentaient les mêmes lieux de prières. La dénégation devient systématique non parce que les appareils policiers sont sûrs d'eux mais au contraire parce que, tout comme les criminels, ils ne veulent pas laisser de "trace" derrière eux. L'invisibilisation, de stratégie au sein d'un dispositif de quadrillage devient nécessité pratique de "masquer ces crimes" et éventuellement de "conserver les personnes" à titre de futures transactions. Cette surenchère crée comme au Sri Lanka, en Colombie ou en Haïti des situations où les milices privées se multiplient et pratiquent entre elles les mêmes méthodes. On "disparaît" de part et d'autre ${ }^{16}$. Comme le signale Daniel Pécaut de manière plus générale : si au départ les acteurs font de la violence une stratégie, tout se passe comme si au fur et à mesure que ces stratégies s'étendent, et font basculer les repères antérieurs, elles se banalisent et rentrent dans "l'ordre des choses". Les disparitions perdent ici leur dimension de projet stratégique et deviennent une pratique réciproque, ou inversement apparaissent parce que les acteurs anticipent leur possible défaite. En devenant pratique réciproque elles renforcent encore leur "normalité" et poussent à l'acceptation d'un tel état de fait. Les disparitions participent alors d'une microphysique des pouvoirs où la stratégie n'est plus réservée à un acteur plutôt qu'à un autre et où la violence symbolique qu'elles incluent se généralise. Nous retrouvons là une des raisons de la confusion initiale qui court dans les approches sur les disparitions.

14 Ceci nous amène à un dernier point. La disparition comme pratique au sein du répertoire d'action coercitive est-elle réservée aux gouvernants (et à leurs agences)? Là encore si l'on en reste aux textes juridiques et à leurs interprétations autorisées, la réponse semble positive puisque le seul critère donné pour "caractériser les disparitions" est l'action de l'Etat sur le corps d'une personne contre sa volonté. Mais qu'en est-il des acteurs quasi-étatiques? Que se passe-t-il lorsqu'il y a privatisation des attributs régaliens par un groupe de guérilla sur un territoire donné ? Ces groupes agissent-ils différemment? Qu'il s'agisse de l'UNITA, du Front Polisario, du PKK ou des différents mouvements qui ont une stratégie de reconnaissance par des instances internationales, il semble que soumis aux mêmes contraintes de contexte, ils agissent de la même manière que les gouvernants lorsqu'ils tiennent à contrôler leur population et à mettre en place un dispositif de quadrillage empêchant l'émergence d'alternative politique à leur mouvement. Mais nos connaissances sur ce sujet des pratiques de disparition dans les zones contrôlées depuis longtemps par ces mouvements est encore balbutiante puisque les ONG ne considéraient pas jusqu'au début des années 1990 que ceci entrait dans leur mandat. Seuls certains récits de prisonniers échappés à des camps de rééducation ou les déclarations de dirigeants en rupture avec l'organisation semblent étayer ce qui reste une hypothèse. Nous reviendrons sur ce point dans un futur numéro. Disparition comme résultante de l'ignorance de l'observateur, disparition comme projet stratégique et comme pratique au sein d'un répertoire d'action, disparition comme produit de la relation de pouvoir entre acteurs en concurrence. Ces trois dimensions sont sans cesse superposées, imbriquées dans l'usage courant du terme et dans les procédures de recensement statistique. Mais ne faut-il pas se distancier de ce nominalisme et de ce positivisme juridique ? Ce qui est au coeur du processus de disparition tient sans doute à ce qu'elle apparaît, à la différence peut-être des pratiques d'exécutions extrajudiciaires ou de tortures, non seulement comme une 
pratique coercitive mais aussi comme une pratique de violence symbolique qui, par le jeu sur l'invisible, le silence, l'incertitude, la complicité objectivée va au delà de l'individu pour frapper les relations sociales.

Coercition et violence symbolique Coercition et violence symbolique : les disparitions mêlent donc intimement ces deux dimensions trop souvent analysées comme opposées ou, à tout le moins, la seconde étant perçue comme une euphémisation de la première. On ne peut pourtant opposer une véritable violence (qui serait la violence physique) à une fausse violence (qui serait la violence symbolique). Ce genre de coupure fortement positiviste et qui croit pouvoir s'appuyer sur la "matérialité des faits" prend en réalité partie pour celui des acteurs qui est en position de domination et qui a les moyens de maintenir le statu-quo en utilisant la langue de la diplomatie comme arme dans le combat $^{17}$. Le choix de tracer une frontière dans le continuum des pratiques de violence n'est en effet pas neutre. En excluant du champ d'analyse les luttes de violence symbolique, on exclut dans le même mouvement de clôture, la relation de l'observateur à l'objet et le fait que les discours sur la violence peuvent être des éléments dans les luttes de pouvoir symbolique, dans les enjeux de légitimation et les luttes de labellisations. Une analyse globale des formes de la violence doit donc inclure l'étude de la violence symbolique et intégrer à la connaissance des mécanismes de violence, les effets que produisent les discours comme pouvoir symbolique (y compris les discours "savants"). Le discours (la parole) ne s'oppose pas forcément à la violence (geste physique) comme on le croit trop souvent dans nos sociétés. La démocratie ne peut pas exclure la violence comme l'a souligné depuis longtemps David Apter. Ceci peut paraître choquant à ceux qui veulent absolument croire à la véracité des textes fondateurs des régimes constitutionnels opposant la violence (barbare) au discours (de l'agora) mais ne s'agit-il pas justement que d'un effet de croyance? La violence fait partie de nos pratiques sociales, y compris dans les Etats de droit, et la violence symbolique y a une place de choix.

16 La violence symbolique n'est pas non plus simplement un moyen de supprimer l'usage de la coercition, une euphémisation heureuse des pratiques de violence physique qui réduirait globalement le niveau de violence dans la société. La "pacification des moeurs" dont parle Elias n'est pas tant une réduction de la violence et une pacification au sens de réconciliation qu'un transfert des pratiques de violence physique vers des formes de violence symbolique dont rien ne nous dit qu'elles soient une forme de "progrès", de "civilisation" comme certaines lectures rapides de l'ouvrage le laissent penser ${ }^{18}$. La violence symbolique ne conduit pas à la paix sociale et ne met pas forcément fin à la violence physique. Cette dernière est toujours vécue comme retenue, suspendue et prête à éclater. La violence symbolique redouble donc souvent la violence physique. Parfois, rarement, les dynamiques d'interdépendances sociales favorisent le registre de la violence symbolique par le biais de l'auto-contrainte, moins directement visible et poussent certes à une raréfaction du registre de la violence physique mais le plus souvent il n'y a que superposition et combinaison des registres. Si le processus d'interdépendance sociale ne se développe pas, la violence physique reste quasiment au même niveau alors même que la violence symbolique s'accroit. La trajectoire historique occidentale reste donc à cet égard exceptionnelle.

17 Par ailleurs, la "civilisation" du nucléaire et de la gestion de la menace d'Apocalypse n'est pas forcément "moins" violente que celle de l'affrontement du "guerrier sauvage". La "civilisation" de l'intériorisation de la peur du crime n'est pas non plus 
moins violente que celle de la cour des miracles. La "civilisation" du contrôle digitalisé et des technologies de surveillance ne l'est pas moins que celle des supplices. Il s'agit plutôt d'autres modalités où la violence s'inscrit dans le langage, les processus d'économie psychique des individus et les relations entre eux, d'autres dispositifs où le quadrillage et le contrôle s'ajoutent à l'inscription sur les corps, bref à d'autres formes de gouvernementalité pour reprendre la terminologie de Foucault. En revanche, il faut peut-être douter de l'irréductibilité des deux formes de l'énoncable et du visible, du savoir et du pouvoir. Foucault exagère peut-être la différence entre les forces et les formes. Celles-ci ne sont pas forcément hétérogènes, distinctes et mélangées simplement par leur capture réciproque dans les dispositifs. Elles se confondent et fusionnent dans la violence symbolique ${ }^{19}$ qui git dans la force des formes. Le pouvoir ne passe donc peut-être pas simplement par des forces mais aussi par ces formes qui ont leur propre force ${ }^{20}$. Quel rapport le discours entretient-il alors avec le pouvoir ? Est-ce que tout discours est une forme de violence symbolique? Potentiellement oui, dès que la relation met en rapport des acteurs en position asymétrique, et ayant un capital symbolique différent mais elle n'est rendue visible que dans son exercice, lorsque le discours suppose la "complicité" de l'agent social dominée ${ }^{21}$, c'est à dire lorsqu'il y a ajustement des catégories de perception de cet agent aux déterminants de sa position, lorsqu'il accepte comme "vrai", "inquestionnable" le discours de pouvoir ${ }^{22}$. La notion de capital symbolique permet de penser ensemble les forces et les formes, le pouvoir et le savoir, les objectivations et les énoncés. Le discours politique, le discours juridique ne sont pas les discours du quotidien en ce qu'ils sont porteurs de violence symbolique. Ici le texte de David Apter nous montre à propos de la Chine et de Mao à quel point le pouvoir peut s'exercer à travers une mise en forme, une mise en sens, un discours producteur de violence symbolique qui "unifie" le sens de l'Histoire et rend inquestionnable sa "vérité". Il analyse comment Mao s'est auto-institué comme narrateur du texte historique révolutionnaire, comment le mythe de Yan'an l'a conduit au pouvoir et lui a permis d'y rester en faisant admettre que la communauté morale se devait d'user de coercition pour s'établir et se perpétuer. Il ne s'agit pas simplement de stratégies de légitimation de pratiques de pouvoir comme une lecture rapide le laisserait supposer mais d'un discours comme pouvoir, d'un discours qui par ses formes ajoute sa force à la force et rend possible un déséquilibre des forces en faveur de celui qui l'énonce. En cela l'approche de David Apter est neuve. Elle permet de penser ce qui restait trop disjoint chez Foucault et sans doute trop allusif dans le débat Bourdieu / Boltanski. Elle propose une piste de recherche sur la violence symbolique qu'il faudra sans doute discuter et approfondir de manière critique. Nous nous y emploierons l'année prochaine dans un numéro sur ce problème de la violence symbolique.

\section{NOTES}

1. Nous ne donnons aucun caractère euphémisé ou légitimant à ce terme de coercition. 
2. Arendt (H.), Les origines du totalitarisme, Seuil, Paris, 1984 ; Lefort (C.), un homme en trop, Seuil, Paris, 1976 ; Fein (H.), Génocide, a sociological perspective, Sage

Publications, 1993 ; Todorov (T.), Face à l'extrême, Seuil, 1991.

3. Gurr (T.), Harff (B.), "Victims of the state : genocides, politicides \& group repression since 1945" in International review of victimology, 1989 /1. Gurr (T.), "Why minorities rebel" in International Political Science Review, 1993/4. Gurr (T.), Minorities at Risk : a global view of ethnopolitical conflicts, USIP Press, Washington 1993.

4. Wolfgang Heinz, Determinants of gross human rights violations, Leiden University, 1992, bibliographie très complète.

5. Amnesty International, Rapport 1993, Efai, 1993, 382 p. ; Rapport, "L'inacceptable"

sur les disparitions et les assassinats politiques, Efai, 1993, 127p. Voir aussi les rapports par pays d'International Alert.

6. Guy Nicolas, "De l'usage des victimes dans les stratégies politiques contemporaines" in Cultures \& Conflits, $n^{\circ}$ 8, hiver 1992.

7. Voir le texte de Francis Perrin pour l'analyse juridique.

8. Voir dénombrer les disparitions : les chiffres enjeux de transactions, voir le texte de Philippe Chapleau sur l'Afrique du Sud.

9. Voir Gilles Deleuze, Capitalisme et schizophrénie : L'anti-oedipe, Les Editions de Minuit, 1972, sur les machine guerrières, leur rapport au socius et au marquage des corps et du territoire, voir sous un angle plus traditionnel la vision Clausewitzienne de la guerre, voir enfin sur les processus de suppression de la position de Tiers, Didier

Bigo, "La conflictualité" in Approches Polémologiques, FEDN, Documentation Française, 1992.

10. Nous verrons plus loin les cas spécifiques dans lesquels les disparitions comme "stratégies" s'intégrent dans ce dispositif.

11. Voir le texte de Daniel Hermant.

12. Ce genre de stratégie est plus fréquent comme point d'application d'un dispositif de quadrillage mais il peut aussi bien sûr motiver des pratiques lors de guerres civiles 13. A l'échelle interne, des secteurs "modernistes" peuvent ainsi "ignorer" la situation ou refuser de la voir. A l'échelle internationale, les gouvernements des Etats de droit peuvent minimiser si nécessaire les protestations des ONG.

14. De plus en plus on remarque la création d'ONG "humanitaire" instrumentalisées par ces Etats pour contredire les ONG occidentales et brouiller les cartes.

15. N'oublions pas non plus la présence d' instructeurs américains, français à leurs côtés et le fait que ces régimes se justifient par référence aux techniques contreinsurrectionnelles de la guerre d'Algérie ou du Viet-Nam.

16. Voir les textes d'Eric Meyer et Daniel Pécaut.

17. Nous nous distançons sur ce point de l'analyse de Philippe Braud, "la violence politique repères et problèmes" in La violence politique dans les démocraties européennes occidentales, ouvrage collectif sous la direction de Philippe Braud, L'Harmattan, 1993.

18. Voir par exemple la lecture de Jean Claude Chesnais dans Histoire de la violence, Robert Laffont, collection pluriel, 1989, ou de Sébastian Roché dans Le sentiment d'insécurité, PUF, 1993, ainsi que d'autres lectures qui glissent de l'évolutionnel vers l'évolutionniste, confondent Elias et Tocqueville et font du premier un "progressiste". 19. Nous prenons ici le contre pied de l'analyse de Foucault. Cf. Gilles Deleuze, Foucault, Les Editions de Minuit, 1986. 
20. Sans doute Pierre Bourdieu dans Ce que parler veut dire, Fayard, 1982, le montre-til et remet-il en cause la distinction foucaldienne.

21. Voir Pierre Bourdieu sur la notion de violence symbolique in Réponses, Seuil, 1992, p. 142 et suivantes.

22. Les conditions d'énonciation des discours, la position de leurs locuteurs sont fondamentales à étudier. Mais si le discours questionne ses conditions d'origine, s'il est critique et auto critique, ou s'il met en relation de face à face des individus ayant approximativement la même position, y a-t-il forcément exercice d'une violence symbolique ? On peut en douter. Toute parole n'est pas forcément violence symbolique car toute logique d'argumentation, de critique et d'organisation du monde n'est pas nécessairement le produit d'un rapport de force, d'une lutte d'intérêts. La discussion est aussi lieu de production de l'accord comme le signale Luc Boltanski et Laurent Thévenot dans L'économie des grandeurs, . En laissant entendre le contraire, Pierre Bourdieu semble généraliser à un point tel la violence symbolique qu'il ne peut plus en donner lui-même les conditions d'émergence et l'historicité. Il finit par la "naturaliser".

INDEX

Mots-clés : disparitions, répression, sciences politiques, violence, invisible 